\title{
Structural Modification of Pristine Graphene Network Towards Nanoporous Graphene Membrane: A Review
}

\author{
Mohd 'Azizir-Rahim Mukria, Mohd Syafiq Elias', Madzlan Aziz', Masaki \\ Tanemura $^{\mathrm{c}}$, Mohd Zamri Mohd Yusop ${ }^{\mathrm{a}^{*}}$ \\ ${ }^{a}$ Faculty of Mechanical Engineering, Universiti Teknologi Malaysia, \\ 81310 UTM Johor Bahru, Johor, Malaysia \\ ${ }^{\mathrm{b}}$ Faculty of Science, Universiti Teknologi Malaysia, \\ 81310 UTM Johor Bahru, Johor, Malaysia \\ ${ }^{\mathrm{c}}$ Department of Physical Science and Engineering, Nagoya Institute of Technology, \\ Gokiso-cho, Showa-ku, Nagoya 466-8555, Japan \\ Submitted: 3/5/2018. Revised edition: 23/5/2018. Accepted: 28/5/2018. Published online: 4/6/2018
}

\begin{abstract}
A single graphene layer is superior many ways preferably in electronic devices application. However, mild modification of the graphene network could open a new potential to the ultrathin graphene membrane. Moreover, recent studies demonstrated that a few simple techniques could generate and control the nanopores size on single layer graphene sheet simultaneously. This review paper will discuss all potential techniques that are capable to generate nanopores structure on the pristine single layer graphene network.
\end{abstract}

Keywords: Graphene, ion irradiation, etching, transmission electron microscope, flux rate

\subsection{INTRODUCTION}

Recently, researchers set an excessive attention on the ability of carbon nanomaterials to act as based structure of membranes for ion separation [1-2]. Currently, a few research works on the improvement of water permeability by molecular filtering medium of polymers [3-7], ceramics [7-11] and organic molecules [12-15]. Even though these materials are theoretically effective, the practical applications for these mediums are not yet fully explored.

Carbon nanomaterials-based membranes materials are challenging to shape in a cost effective and mass production. Moreover, these membranes show ineffective with regard to the exclusion of salt ions, as well as indicating a stubby water flux hence proving the requirement of ultrathin and low cost membranes [16$20]$.

World demand on environmental solution of water crisis led to the novel discoveries of new classes of nanomaterials. Three decades ago, twodimensional (2D) materials were considered non-existent materials which only applicable in fundamental theory before the discovery of graphene, a single atomic layer of carbon lattice arranged in a honeycomb like structure. Interestingly, most of the researchers believed that $2 \mathrm{D}$ materials could not exist or predominantly stable due to thermodynamic instabilities in a free state [21-22]. However in 2004, Nobel Laureates Geim and Novoselov demonstrated the novel exfoliating process which extract a $2 \mathrm{D}$ materials from bulk structures verifying the isolation of graphene [23]. 
Graphene are exceptional in many ways and eventually became trending materials which motivated more exploration prior the creation of $2 \mathrm{D}$ layers from a stacking $3 \mathrm{D}$ bulk material [24-25]. In contrast, there are also different type of 2D graphene-like structure such as hexagonal $\mathrm{BN}$ (hBN) [26], transition metal oxides, carbide and carbonitrides, [27-32] or the most recent discovery, silicene (silicon-based) or germanene (germanium-based) as a 2D materials [33-34]. These 2D nanostructures indicating a similar properties to the graphene properties such as atomic thickness, surface area (typically over $2500 \mathrm{~m} 2 \mathrm{~g}-1)$ [35], strength $(\sim 2.4 \pm 0.4$ TPa) [36-38], exciting durability, and hydrophobicity signifying that these materials too could act as effective water filtration membranes. Still, this area awfully requires significant development.

Graphene is superior on its atomically thin structure and large surface area; hence graphene is ideal to be considered as ultrathin separation membrane which high selectivity molecules through the carbon network. However, graphene is impenetrable in its pristine crystalline form to all gases including helium and all type of liquids [39-40].

Regardless of the impassable nature of graphene, there are enormous interest to tune the ultrathin crystalline structure of graphene by introducing nanopores converting graphene as ultimate separation membrane [41-42]. However, the method to produce uniform size nanopores are extremely challenging and difficult due to graphene large surface area. [2, 43-44]. There were a few reports demonstrating experimental and dynamic simulation studies challenging in creating subnanometer pores structure on graphene network using methods such as ion bombardment, oxidation and electron beam irradiation [1, 45-47]. In this review, the recent techniques to install nanopores structure on the pristine single layer graphene network were deeply explored. These techniques were briefly discussed in term of effectiveness, tendency of defects and possibility of the porosity to be tunable.

\subsection{ION BOMBARDMENT AND PLASMA ETCHING}

One of the potential technique to create nanopores structure on graphene is ion bombardment. O'Hern et al. demonstrated low energy of ion bombardment and simultaneous chemical oxidation potential to create atomic scale nanopores on monolayer graphene [48]. These atomic scale nanopores were produced on macroscale graphene thin film that synthesized using CVD and followed by ion bombardment. The subnanometer pores were further etched using chemical oxidation. In this work, O'Hern et al. also discussed that etching time has a significant effect on the controlling the pores density. The pores density gradually increased from $0-6 \times 10^{-12} \mathrm{~cm}^{-2}$ by the increment of etching time from $0-120$ minutes and stopped when it reached 60 minutes of etching time as showed in Figure 1. This significant result indicating the possibilities of graphene as selective membrane on transit or rejection of ions and any organic compounds. However, the excessive etching subsequently creates more defects and damaging the graphene network hence resulting poor the flux rate. This technique is also only limited for moderate pore density which limiting the selectivity compound sizes. In other report, O'Hern et al. demonstrated graphene defect modification via ion irradiation [49]. The relatively defect free graphene shows significant results in rejecting up 
to $90 \%$ of residual molecules and the flux rate was comparable with the dynamic simulation results. However, salt molecules were transported over the membrane at a flux rate higher than water. Other findings observed by Surwade et al. emphasizing significantly low water molecules passed through graphene membranes, variance to the results after oxygen plasma treatment, regardless of similar peaks in the Raman spectra as shown in Figure 2 [50].
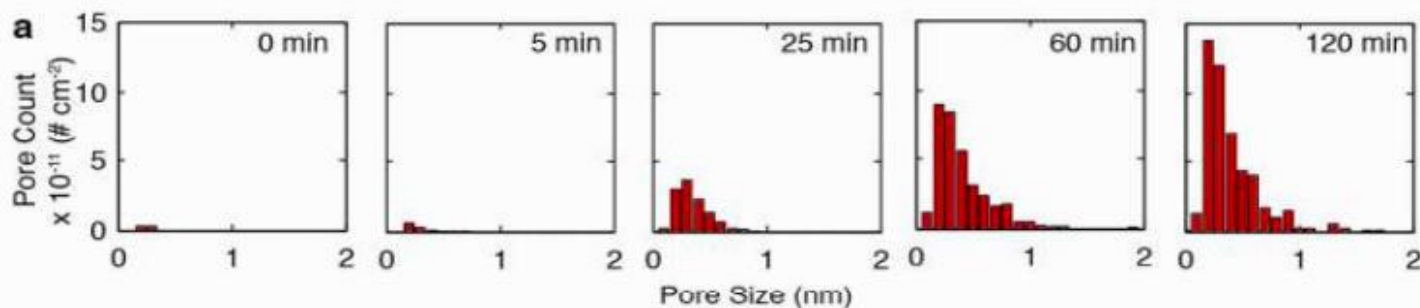

b

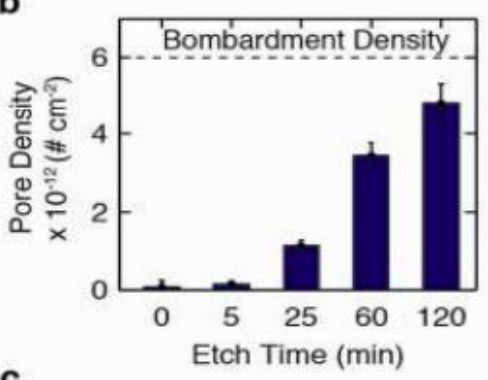

d
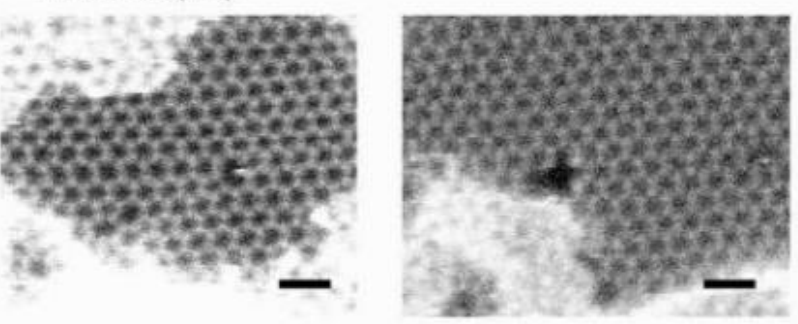

C
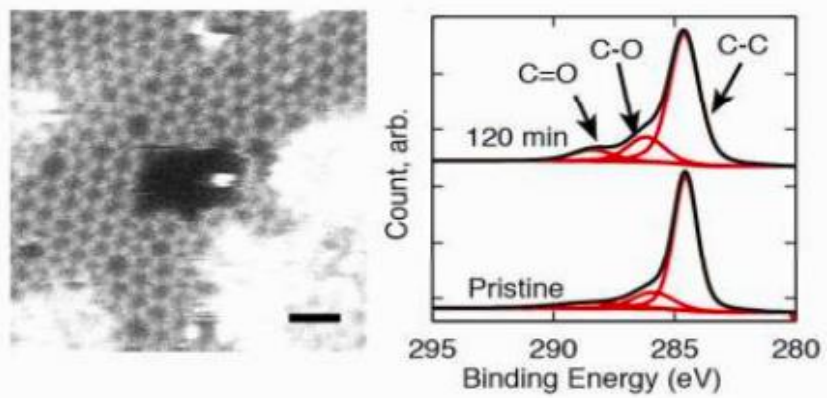

Figure 1 Characterization of pores in graphene network created though ion irradiation followed by chemical oxidation; (a) Distribution of pore sizes at $0 \mathrm{~min}, 5 \mathrm{~min}, 25 \mathrm{~min}, 60 \mathrm{~min}$, and 120 min etch times, (b) Pore density etched at $120 \mathrm{~min}$, (c) the growth of mean pore diameter with etched time, (d) Nanopores observed using HRTEM for monovacancy, $0.5 \mathrm{~nm}$ diameter pore, and $1 \mathrm{~nm}$ diameter pore [49]

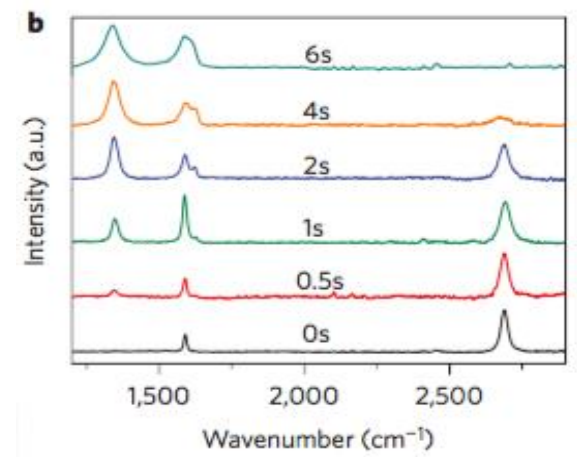

Figure 2 Raman spectra of graphene at difference oxygen plasma exposure times [50]
The irradiation by ions of different energies were applied within range 250 $\mathrm{V}$ to $20 \mathrm{kV}$ and by gallium ions at 30 $\mathrm{kV}$. Normally, the main function of ion bombardment was to eject any reactive defects in graphene, then gradually grow into larger pores upon etching time [48, 51-52]. Jang et al. explained that without ion bombardment, water permeance of membranes were poorer at least by a factor of 2 , signifying that the simultaneous treatment of ion 
irradiation and plasma etching amplify higher water transport [53].

Neglecting the ion bombardment treatment, Xie et al. performed hydrogen plasma etching to the graphene strip for nanoelectronics application [54]. There was no visible etched hole formation on graphene network observed by AFM, signifying that selective hydrogen plasma etching only occurred at the graphene edges as shown in Figure 3.

\section{(a)}

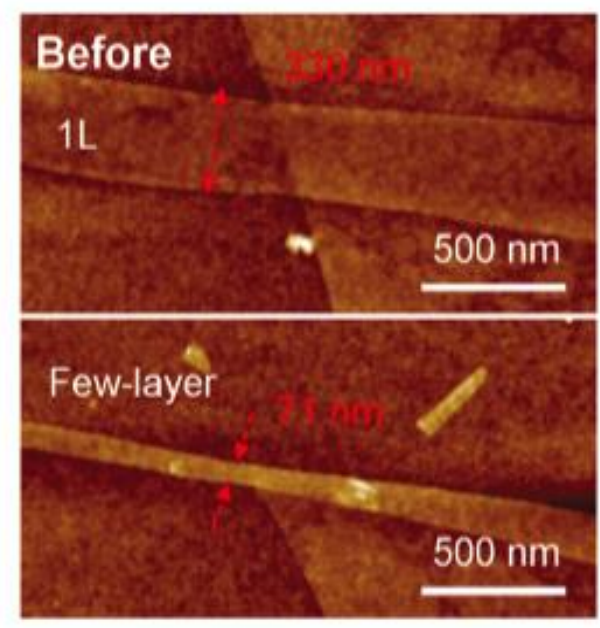

Undeniably, the fabrication of single layer graphene at large area with defectfree are the most challenging task faced in the development of graphene based membranes [44, 55-56]. Although chemical oxidation etching has demonstrated as an alternative for large scale graphene production yet the main problem still unsolved as the various defects found in graphene $[42,57]$.

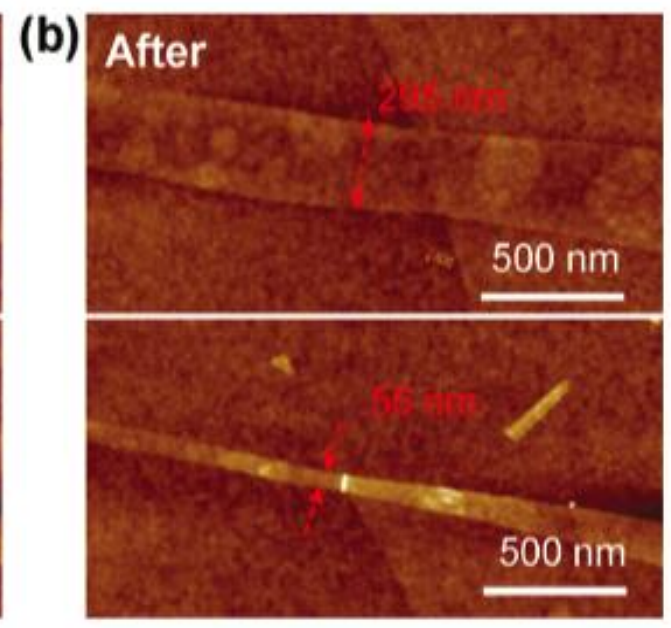

Figure 3 AFM images of graphene [top: a single layer $(1 \mathrm{~L})$ graphene sheet; bottom: a fewlayers graphene sheet] (a) before and (b) after selective H plasma edge etching [54]

\subsection{ION IRRADIATION AND ELECTRON BEAM}

Efforts have been made to develop a reliable technique to fabricate ultrathin graphene-based membranes. Wei et al. demonstrated a double layers graphene synthesized in CVD with minimal defects [58], successively onto a 49 pore puncture on the SiNx frame which the freestanding graphene layers merely $1 \mathrm{~nm}$ thick [59]. The atomic-scale nanopores were precisely formed via a drilling technique using a focused ion beam (FIB). This physically punctured double-layer graphene possessed of random narrowly scattered nanopores which capable of providing ultimate permeation, exhibiting water flux rates in agreement with the 2D materials. Fischbein and Drndic' proved that the nanopores can be generated in multilayer graphene network by the controlled exposure time to a focused electron-beam bombardment using a TEM at ambient temperature (Figure 4) [45].

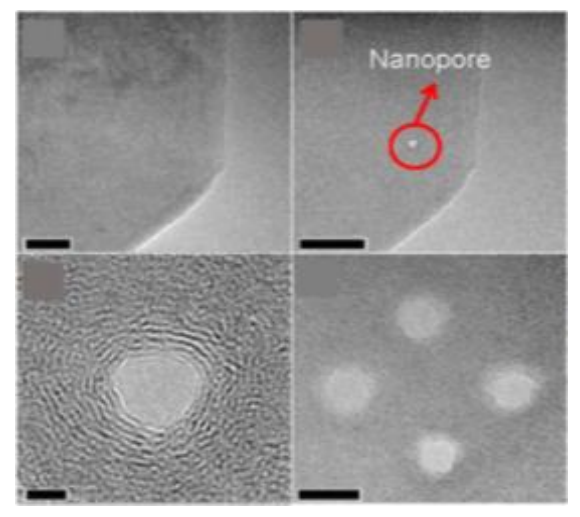

Figure 4 TEM images before and after the nanopore generation on the graphene network by electron-beam bombardment [45] 
In this technique, uniform nanopores were generated in a graphene network within a few seconds. The electron energy are require to be more than 100 $\mathrm{keV}$ to effectively generate the nanopores. Even though there are a few techniques to synthesize the graphene thin film, the vital problem still occurred on the controlling the formation of nanopores with precise sizes. Ion bombardment or focused ion beam could generate precise nanopores size on graphene network, yet this technique is not practical in the industrial field for mass production.

\subsection{HYDROGEN ETCHING}

As CVD can offers $\mathrm{H}_{2}$ etching, it has become favorable for current researcher to explore and validate the effectiveness and efficiency towards fabricating nanoporous graphene membranes for seawater desalination. This technique has become prominent in order to avoid contamination since it can perform continuously after graphene synthesized process. In addition, this technique provides natural etching treatment without inducing high defect as it only involve $\mathrm{C}$ detachment, which is reflecting reverse reaction of graphene growth. As mention earlier, alternative techniques such as oxygen or hydrogen plasma would induce nonuniform nanoporous distribution besides low coverage across the growth graphene [50].

Etching at $800{ }^{\circ} \mathrm{C}$ in 30 minutes, Zhang et al. found highly anisotropic etching with $80 \%$ are 1200 angle of graphene edges [61]. This proving that continuous graphene can be etched to have numerous hexagonal holes as shown in Figure 5. The etching reaction will be accelerated after small openings were etched on graphene. The $\mathrm{Cu}$ substrate underneath the graphene would act as a catalyst for hydrogen to react with carbon atoms in graphene network.

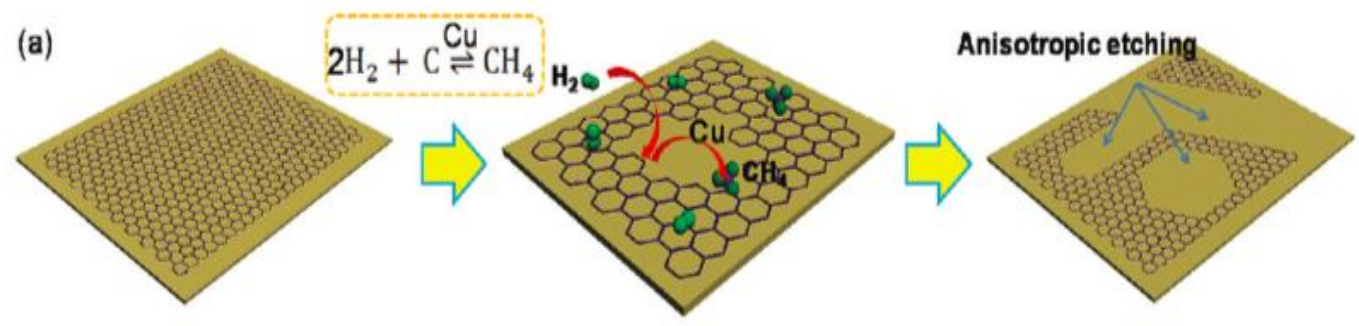

Figure 5 Schematic diagram of H2 etching mechanism [61]

The edge of the etched holes became almost circle-shape, indicating a reduction of the anisotropic property of the etching once the etching temperature raised up to $1000{ }^{\circ} \mathrm{C}$. In the opposite, there is no etching reaction when the temperature decreased lower than $600{ }^{\circ} \mathrm{C}$. Theoretically, the etching will only happen when the temperature reaches a certain point to overcome the activation energy to unchain the $\mathrm{C}-\mathrm{C}$ bonds in graphene network.
Separately, Wang et al. revealed that etched frequencies are correlated in density and width with time. Hence, at the high etching percentage of graphene, the formation of graphene islands in the single graphene domain was observed [62]. The hexagonal domain shape was preserved over the large domain sizes and the graphene lattice structure was not deformed by etching. When the etching time was further extended to $360 \mathrm{~min}$, the single graphene structure 
was etched away as shown in Figure 6. The trough formation was believed formed due to cooling process after graphene growth, which might have caused the wrinkle formation on the graphene thin film on $\mathrm{Cu}$ substrate given the large thermal expansion coefficient difference between both the graphene and the $\mathrm{Cu}$.
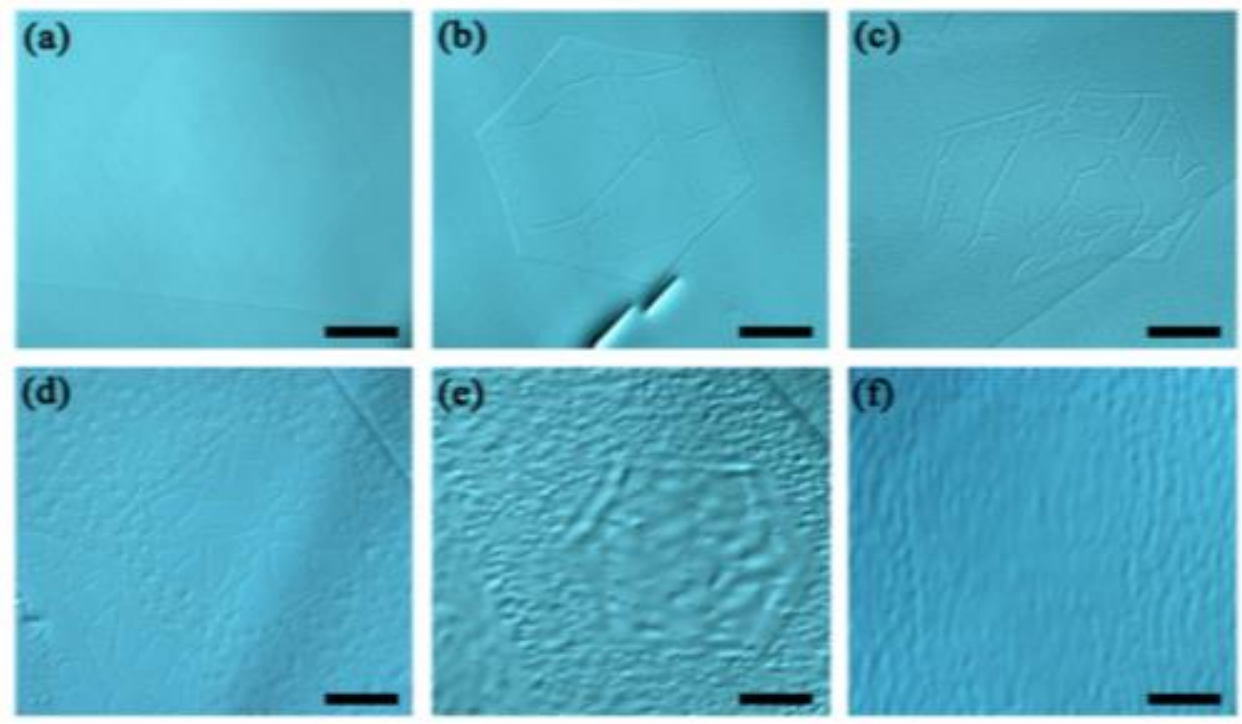

Figure 6 (a) Optical microscope images of graphene before etching, (b-f) Optical microscope images of the degree of etching process depending on the etching time of 7,20,60,240, and $360 \mathrm{~min}$. (The scale bar is $20 \mu \mathrm{m}$ ) [62]

\subsection{CONCLUSION}

Several types of recent modification of pristine graphene have been highlighted to become nanoporous graphene. It is believed that all these graphene membranes are fabricated as a selective membrane for water purification and gas separation. There are still many challenges to be tackled before such superior membranes could be of practical use. These include good mechanical stability under high flow rates and pressure, fabrication of supporting frame of large continuous sheets of graphene as a membrane module and uniform nanopore generation in the graphene network. More improvements in theoretical point of view and technique to support the practical application of nanoporous graphene membranes are still required. Moreover, large area defect-free nanoporous graphene membranes with high density subnanometer pores are preferable in the industrial application. Thus, the large-area defect-free graphene fabrication, defect free delicate graphene transfer and uniform nanopore generation methods should be paid more attentions.

\section{ACKNOWLEDGEMENT}

This work was financially supported by Universiti Teknologi Malaysia (UTM) under UTM Zamalah Scholarship, HICoE grant (R.J090301.7824.4J202), Flagship grant (Q.J130000.2424.03G36), GUP Tier 1 grant (Q.J130000.2524.16H37) and Ministry of Higher Education Malaysia (MOHE). 


\section{REFERENCES}

[1] T. Humplik, J. Lee, S. C. O'Hern, B. A. Fellman, M. A. Baig, S. F. Hassan, M. A. Atieh, F. Rahman, T. Laoui, R. Karnik and E. N. Wang. 2011. Nanostructured Materials for Water Desalination. Nanotechnology. 22(29): 292001.

[2] K. A. Mahmoud, B. Mansoor, A. Mansour and M. Khraisheh, 2015, Functional Graphene Nanosheets: the Next Generation Membranes for Water Desalination. Desalination. 356: 208-225.

[3] M. Ulbricht. 2006. Advanced Functional Polymer Membranes. Polymer. 47(7): 2217-2262.

[4] W. J. Lau, A. F. Ismail, N. Misdan and M. A. Kassim. 2012. A Recent Progress in Thin Film Composite Membrane: A Review. Desalination. 287: 190-199.

[5] G. M. Geise, D. R. Paul and B. D. Freeman. 2014. Fundamental Water and Salt Transport Properties of Polymeric Materials. Progress in Polymer Science. 39(1): 1-42.

[6] J. Dulebohn, P. Ahmadiannamini, T. Wang, S. S. Kim, T. J. Pinnavaia and V. V. Tarabara. 2014. Polymer Mesocomposites: Ultrafiltration Membrane Materials with Enhanced Permeability, Selectivity and Fouling Resistance. Journal of Membrane Science. 453: 478-488.

[7] H. Dong, L. Zhao, L. Zhang, H. Chen, C. Gao and W. W. Ho. 2015. High-flux Reverse Osmosis Membranes Incorporated with Nay Zeolite Nanoparticles for Brackish Water Desalination. Journal of Membrane Science. 476: 373-383.

[8] M. Safarpour, A. Khataee and V. Vatanpour. 2015. Thin Film Nanocomposite Reverse Osmosis Membrane Modified by Reduced
Graphene oxide/TiO2 with Improved Desalination Performance. Journal of Membrane Science. 489: 43-54.

[9] J.-W. Zhang, H. Fang, J.-W. Wang, L.-Y. Hao, X. Xu and C.S. Chen. 2014. Preparation and Characterization of Silicon Nitride Hollow Fiber Membranes for Seawater Desalination. Journal of Membrane Science. 450: 197-206.

[10] A. Garofalo, L. Donato, E. Drioli, A. Criscuoli, M. C. Carnevale, O. Alharbi, S. A. Aljlil and C. Algieri. 2014. Supported MFI Zeolite Membranes by Cross Flow Filtration for Water Treatment. Separation and Purification Technology. 137: 2835.

[11] J. Kujawa, S. Cerneaux, S. Koter and W. Kujawski. 2014. Highly Efficient Hydrophobic Titania Ceramic Membranes for Water Desalination. ACS Applied Materials \& Interfaces. 6(16): 14223-14230.

[12] X. Li, S. Chou, R. Wang, L. Shi, W. Fang, G. Chaitra, C. Y. Tang, J. Torres, X. Hu and A. G. Fane. 2015. Nature Gives the Best Solution for Desalination: Aquaporin-based Hollow Fiber Composite Membrane with Superior Performance. Journal of Membrane Science. 494: 68-77.

[13] C. Y. Tang, Y. Zhao, R. Wang, C. Hélix-Nielsen and A. G. Fane. 2013. Desalination by Biomimetic Aquaporin Membranes: Review of Status and Prospects. Desalination. 308: 34-40.

[14] Y. X. Shen, W. Si, M. Erbakan, K. Decker, R. D. Zorzi, P. O. Saboe, Y. J. Kang, S. Majd, P. J. Butler, T. Walz, A. Aksimentiev, J. L. Hou, M. Kumar and D. A. Weitz. 2015. Highly Permeable 
Artificial Water Channels that Can Self-assemble Into TwoDimensional Arrays. Proceedings of the National Academy of Sciences. 112(32): 9810-9815.

[15] M. M. Pendergast and E. M. Hoek. 2011. A Review of Water Treatment Membrane Nanotechnologies. Energy \& Environmental Science. 4: 19461971.

[16] F. Fornasiero, J. B. In, S. Kim, H. G. Park, Y. Wang, C. P. Grigoropoulos, A. Noy and O. Bakajin. 2010. PH-tunable Ion Selectivity in Carbon Nanotube Pores, Langmuir. 26(18): 1484814853.

[17] F. Fornasiero, H. G. Park, J. K. Holt, M. Stadermann, C. P. Grigoropoulos, A. Noy and O. Bakajin. 2008. Ion Exclusion by sub-2-nm Carbon Nanotube Pores. Proceedings of the National Academy of Sciences. 105(45): 17250-17255.

[18] Z. Hu, Y. Chen and J. Jiang. 2011. Zeolitic Imidazolate Framework8 as a Reverse Osmosis Membrane for Water Desalination: Insight from Molecular Simulation. The Journal of Chemical Physics. 134(13): 134705.

[19] C. H. Cho, K. Y. Oh, S. K. Kim, J. G. Yeo and P. Sharma. 2011. Pervaporative Seawater Desalination using NaA Zeolite Membrane: Mechanisms of High Water Flux and High Salt Rejection. Journal of Membrane Science. 371(1-2): 226-238.

[20] L. Lai, J. Shao, Q. Ge, Z. Wang and Y. Yan. 2012. The Preparation of Zeolite $\mathrm{NaA}$ Membranes on the Inner Surface of Hollow Fiber Supports. Journal of Membrane Science. 409: 318-328.
[21] R. Peierls. 1935. Statistical Error in Counting Experiments. Proceedings of the Royal Society of London. Series A. Mathematical and Physical Sciences. 149(868): 467-486.

[22] L. D. Landau. 1937. Zur Theorie der phasenumwandlungen II. Phys. Z. Sowjetunion. 11(545): 26-35.

[23] K. S. Novoselov, A. K. Geim, S. V. Morozov, D. Jiang, Y. Zhang, S. V. Dubonos, I. V. Grigorieva and A. A. Firsov. 2004. Electric Field Effect in Atomically Thin Carbon Films. Science. 306(5696): 666-669.

[24] K. Novoselov, A. K. Geim, S. Morozov, D. Jiang, M. Katsnelson, I. Grigorieva, S. Dubonos and A. Firsov. 2005. Two-dimensional Gas of Massless Dirac Fermions in Graphene. Nature. 438(7065): 197-200.

[25] A. K. Geim and K. S. Novoselov. 2007. The Rise of Graphene, Nature Materials. 6(3): 183-191.

[26] D. Pacile, J. Meyer, C. O. Girit and A. Zettl. 2008. The Twodimensional Phase of Boron Nitride: $\quad$ Few-Atomic-Layer Sheets and Suspended Membranes. Applied Physics Letters. 92: 133107.

[27] J. N. Coleman, M. Lotya, A. O’Neill, S. D. Bergin, P. J. King, U. Khan, K. Young, A. Gaucher, S. De, R. J. Smith, I. V. Shvets, S. K. Arora, G. Stanton, H. Y. Kim, K. Lee, G. T. Kim, G. S. Duesberg, T. Hallam, J. J. Boland, J. J. Wang, J. F. Donegan, J. C. Grunlan, G. Moriarty, A. Shmeliov, R. J. Nicholls, J. M. Perkins, E. M. Grieveson, K. Theuwissen, D. W. McComb, P. D. Nellist and V. Nicolosi. 2011. Two-dimensional Nanosheets Produced by Liquid Exfoliation 
of Layered Materials. Science. 331(6017): 568-571.

[28] R. Ma and T. Sasaki. 2010. Nanosheets of Oxides and Hydroxides: Ultimate 2D ChargeBearing Functional Crystallites. Advanced Materials. 22(45): 5082-5104.

[29] M. Chhowalla, H. S. Shin, G. Eda, L.-J. Li, K. P. Loh and H. Zhang. 2013. The Chemistry of Twodimensional Layered Transition Metal Dichalcogenide Nanosheets. Nature Chemistry. 5(4): 263-275.

[30] C. Ataca, H. Sahin and S. Ciraci. 2012. Stable, Single-layer $m x 2$ Transition-metal Oxides and Dichalcogenides in a Honeycomb-like Structure. The Journal of Physical Chemistry $C$. 116(16): 8983-8999.

[31] M. Naguib, O. Mashtalir, J. Carle, V. Presser, J. Lu, L. Hultman, Y. Gogotsi and M. W. Barsoum. 2012. Two-dimensional Transition Metal Carbides. ACS Nano. 6(2): 1322-1331.

[32] M. Naguib, M. Kurtoglu, V. Presser, J. Lu, J. Niu, M. Heon, L. Hultman, Y. Gogotsi and M. W. Barsoum. 2011. Twodimensional Nanocrystals Produced by Exfoliation of $\mathrm{Ti}_{3} \mathrm{AlC}_{2}$. Advanced Materials. 23(37): 4248-4253.

[33] P. Vogt, P. D. Padova, C. Quaresima, J. Avila, E. Frantzeskakis, M. C. Asensio, A. Resta, B. Ealet and G. L. Lay. 2012. Silicene: Compelling Experimental Evidence for Graphenelike Two-dimensional Silicon. Physical Review Letters. 108: 155501.

[34] K. J. Koski and Y. Cui. 2013, The New Skinny in Two-dimensional Nanomaterials. ACS Nano. 7(5): 3739-3743.
[35] M. D. Stoller, S. Park, Y. Zhu, J. An and R. S. Ruoff. 2008. Graphene-based Ultracapacitors. Nano Letters. 8(10): 3498-3502.

[36] C. Lee, X. Wei, J. W. Kysar and J. Hone. 2008. Measurement of the Elastic Properties and Intrinsic Strength of Monolayer Graphene. Science. 321(5887): 385-388.

[37] J.-U. Lee, D. Yoon and H. Cheong. 2012. Estimation of Young's Modulus of Graphene by Raman Spectroscopy. Nano Letters. 12(9): 4444-4448.

[38] P. Zhang, L. Ma, F. Fan, Z. Zeng, C. Peng, P. E. Loya, Z. Liu, Y. Gong, J. Zhang, X. Zhang, P. M. Ajayan, T. Zhu and J. Lou. 2014. Fracture Toughness of Graphene, Nature Communications. 5: 3782

[39] O. Leenaerts, B. Partoens and F. M. Peeters. 2008. Graphene: A Perfect Nanoballoon. Applied Physics Letters. 93: 193107.

[40] J. S. Bunch, S. S. Verbridge, J. S. Alden, A. M. van der Zande, J. M. Parpia, H. G. Craighead and P. L. McEuen. 2008. Impermeable Atomic Membranes from Graphene Sheets. Nano Letters. 8(8): 2458-2462.

[41] M. E. Suk and N. R. Aluru. 2010. Water Transport through Ultrathin Graphene. The Journal of Physical Chemistry Letters. 1(10): 1590-1594.

[42] D. Cohen-Tanugi and J. C. Grossman. 2012. Water Desalination Across Nanoporous Graphene. Nano Letters. 12(7): 3602-3608.

[43] F. Perreault, A. F. de Faria and M. Elimelech. 2015. Environmental Applications of Graphene-based Nanomaterials. Chemical Society Reviews. 44(16): 5861-5896

[44] L. Huang, M. Zhang, C. Li and G. Shi. 2015. Graphene-based Membranes for Molecular Separation. The Journal of 
Physical Chemistry Letters. 6(14): 2806-2815.

[45] M. D. Fischbein and M. Drndić. 2008. Electron Beam Nanosculpting of Suspended Graphene Sheets. Applied Physics Letters. 93(11): 113107.

[46] N. Inui, K. Mochiji, K. Moritani and N. Nakashima. 2010. Molecular Dynamics Simulations of Nanopore Processing in a Graphene Sheet by Using Gas Cluster Ion Beam. Applied Physics A. 98(4): 787-794.

[47] D. Wei, Y. Liu, Y. Wang, H. Zhang, L. Huang and G. Yu. 2009. Synthesis of n-doped Graphene by Chemical Vapor Deposition and Its Electrical Properties. Nano Letters. 9(5): 1752-1758.

[48] S. C. O'Hern, M. S. Boutilier, J.C. Idrobo, Y. Song, J. Kong, T. Laoui, M. Atieh and R. Karnik. 2014. Selective Ionic Transport Through Tunable Subnanometer Pores in Single-layer Graphene Membranes. Nano Letters. 14(3): 1234-1241.

[49] S. C. O'Hern, D. Jang, S. Bose, J.C. Idrobo, Y. Song, T. Laoui, J. Kong and R. Karnik. 2015. Nanofiltration Across Defectsealed Nanoporous Monolayer Graphene. Nano Letters. 15(5): 3254-3260.

[50] S. P. Surwade, S. N. Smirnov, I. V. Vlassiouk, R. R. Unocic, G. M. Veith, S. Dai and S. M. Mahurin. 2015. Water Desalination Using Nanoporous Single-Layer Graphene. Nature Nanotechnology. 10(5): 459-464.

[51] O. Lehtinen, J. Kotakoski, A. V. Krasheninnikov, A. Tolvanen, K. Nordlund, J. Keinonen. 2010. Effects of Ion Bombardment on a Two-dimensional Target: Atomistic Simulations of Graphene Irradiation. J. Phys.
Rev. B: Condens. Matter Mater. Phys. 81(15): 153401.

[52] C. J. Russo and J. A. Golovchenko. 2012. Atom-byatom Nucleation and Growth of Graphene Nanopores. Proc. Natl. Acad. Sci. U. S. A. 109(16): 59535957.

[53] D. Jang, J.-C. Idrobo, T. Laoui and R. Karnik. 2017. Water and Solute Transport Governed by Tunable Pore Size Distributions in Nanoporous Graphene Membranes. ACS Nano. 11(10): 10042-10052.

[54] L. Xie, L. Jiao and H. Dai. 2010. Selective Etching of Graphene Edges by Hydrogen Plasma. $J$. Am. Chem. Soc. 132(42): 1475114753.

[55] G. Liu, W. Jin and N. Xu. 2015. Graphene-based Membranes. Chem. Soc. Rev. 44(15): 50165030.

[56] D.-Y. Koh and R. P. Lively. 2015. Nanoporous Graphene: Membranes at the Limit. Nature Nanotechnology. 10: 385-386.

[57] W. Yuan, J. Chen and G. Shi. 2014. Nanoporous Graphene Materials. Materials Today. 17(2): 77-85.

[58] Y. Wei, J. Wu, H. Yin, X. Shi, R. Yang and M. Dresselhaus. 2012. The Nature of Strength Enhancement and Weakening by Pentagon-heptagon Defects in Graphene. Nature Materials. 11(9): 759-763.

[59] K. Celebi, J. Buchheim, R. M. Wyss, A. Droudian, P. Gasser, I. Shorubalko, J.-I. Kye, C. Lee and H. G. Park. 2014. Ultimate Permeation Across Atomically Thin Porous Graphene. Science. 344(6181): 289-292.

[60] Y. Zhang, Z. Li, P. Kim, L. Zhang and C. Zhou. 2012. Anisotropic Hydrogen Etching of Chemical 
Vapor Deposited Graphene. ACS Nano. 6(1): 126-132.

[61] B. Wang, Y. Zhang, H. Zhang, Z. Chen, X. Xie, Y. Sui, X. Li, G. Yu, L. Hu, Z. Jin and X. Liu. 2014.
Wrinkle-dependent Hydrogen Etching of Chemical Vapor Deposition-grown Graphene Domains. Carbon. 70: 75-80. 